\title{
Revista Colombiana de

\section{El consumo regular de bebidas azucaradas incrementa el perfil lipídico-metabólico y los niveles de adiposidad en universitarios de Colombia}

\author{
Robinson Ramírez-Vélez*, Mónica L. Ojeda, M. Alejandra Tordecilla, \\ Jhonatan C. Peña y José F. Meneses
}

Grupo GICAEDS, Facultad de Cultura Física, Deporte y Recreación, Universidad Santo Tomás, Bogotá, Colombia

Recibido el 2 de febrero de 2015; aceptado el 23 de abril de 2015

Disponible en Internet el 3 de julio de 2015

\section{PALABRAS CLAVE \\ Nutrición; \\ Metabolismo; \\ Estilo de vida; \\ Estado de salud}

\begin{abstract}
Resumen
Objetivo: Examinar si el consumo de bebidas azucaradas se relaciona con alteraciones en el perfil lipídico-metabólico y con marcadores de adiposidad en una muestra de estudiantes universitarios de Colombia.

Métodos: Estudio transversal en 280 voluntarios (73,9\% varones) de 3 universidades de Colombia. El consumo de bebidas azucaradas se obtuvo mediante un cuestionario de frecuencia de ingesta en los últimos 7 días (BEVQ-15) en los dominios: bebidas azucaradas carbonatadas y refrescos de jugo. Se identificó la cantidad y la frecuencia de consumo (nunca, una vez, 2-3 veces $y+4$ veces/sem). Se obtuvieron datos bioquímicos de glucosa, colesterol total, triglicéridos, c-HDL, c-LDL, índice arterial e índice de Castelli. Se calculó el índice lipídico-metabólico según las concentraciones de triglicéridos, c-LDL, c-HDL y glucosa. La circunferencia de cintura, el índice de masa corporal, el índice de adiposidad corporal y el porcentaje de grasa corporal por impedancia bioeléctrica se usaron como marcadores de adiposidad.

Resultados: En el grupo de varones, los participantes que acusaron mayor consumo de bebidas azucaradas (+4 veces/sem) presentaron mayores valores de circunferencia de cintura, porcentaje de grasa corporal, colesterol total, triglicéridos, c-LDL, así como en los índices de Castelli y arterial $(p=N S)$. En mujeres se observó esta relación en el índice de masa corporal, la circunferencia de cintura, el porcentaje de grasa corporal, los triglicéridos y el c-LDL ( $p$ tendencia $<0,05)$. En las categorías más altas de consumo $(2-3$ y +4 veces/sem) se observó una relación inversa con el índice lipídico-metabólico $(\mathrm{p}<0,05)$ tras ajustar por sexo, edad e índice de masa corporal.

Conclusión: El incremento en el consumo de bebidas azucaradas se relacionó con un mayor perfil lipídico-metabólico y con marcadores de adiposidad elevados en universitarios colombianos.

( 2015 Sociedad Colombiana de Cardiología y Cirugía Cardiovascular. Publicado por Elsevier España, S.L.U. Este es un artículo Open Access bajo la licencia CC BY-NC-ND (http://creativecommons.org/licenses/by-nc-nd/4.0/).
\end{abstract}

\footnotetext{
* Autor para correspondencia.

Correos electrónicos: robin640@hotmail.com, robinsonramirez@usantotomas.edu.co (R. Ramírez-Vélez).
} 


\section{KEYWORDS}

Nutrition;

Metabolism;

Lifestyle;

Health status

\section{Regular consumption of sugar-sweetened beverages increases the lipid-metabolic profile and adiposity among university students from Colombia}

\begin{abstract}
Objective: To examine whether the consumption of sugar-sweetened beverages is related to disturbances in the lipid-metabolic profile and markers of adiposity in a sample of university students from Colombia.

Methods: A cross-sectional study was undertaken in 280 volunteers from 3 universities in Colombia. Data for sugar-sweetened beverages consumption were collected using a frequency-intake questionnaire in the last week (BEVQ-15) including carbonated beverages and juices. We also estimated data for quantity and frequency of consumption (never, one time, 2-3 times and $>4$ times per week). Data for biomarkers included total cholesterol, triglycerides, c-HDL, c-LDL, arterial index and Castelli index. Lipid-metabolic index was calculated through serum concentrations of triglycerides, c-HDL, c-LDL and glucose. Waist circumference, body mass index and fat mass percentage were evaluated via bioelectrical impedance and used as markers of adiposity.

Results: Male reported higher sugar-sweetened beverages consumption (> 4 times/wk) and elicited higher values of fat mass, waist circumference, total cholesterol, triglycerides, c-LDL and Castelli and arterial indices $(P=\mathrm{NS})$. These relationships were also observed among women for body mass index, waist circumference, fat mass, triglycerides and C-LDL $(P<.05)$. Furthermore, higher sugar-sweetened beverages consumption categories were negatively associated with lipid-metabolic index $(P<.05)$ after adjustments by sex, age and body mass index.

Conclusion: A higher sugar-sweetened beverages consumption was associated with a major lipid-metabolic profile and also with higher concentrations of adiposity markers among university students from Colombia.

(c) 2015 Sociedad Colombiana de Cardiología y Cirugía Cardiovascular. Published by Elsevier España, S.L.U. This is an open access article under the CC BY-NC-ND license (http://creativecommons.org/licenses/by-nc-nd/4.0/).
\end{abstract}

\section{Introducción}

Recientes evidencias destacan la importancia de la ingesta de bebidas azucaradas y la relación con la obesidad ${ }^{1}$, la diabetes mellitus tipo $2(\mathrm{DM}-2)^{2}$, el síndrome metabólico ${ }^{3}$, la hipertensión arterial ${ }^{4}$, la enfermedad coronaria isquémica ${ }^{5}$ y ciertos tipos de cáncer ${ }^{6}$. En un metaanálisis reciente, Rippe $^{7}$ demuestra que el consumo habitual y los grandes volúmenes de bebidas con alto contenido de sacarosa y jarabe de maíz alto en fructosa se asocian con ganancia de peso, resistencia a la insulina, acumulación de tejido adiposo visceral y grasa ectópica, así como con elevación de los niveles de triglicéridos y colesterol. Similar resultado reportaron Malik et al. ${ }^{8}$ en otro metaanálisis que incluyó 11 estudios de cohorte prospectiva, en el que mostraron que el consumo de $334 \mathrm{~mL} /$ día de bebidas azucaradas se asoció con el desarrollo de obesidad (RR 1,20; IC 95\% 1,02-1,42) y DM-2 (RR 1,25; IC 95\% 1,10-1,42). En América Latina, México ilustra claramente la dimensión de este problema, siendo hoy el segundo país que más bebidas azucaradas consume en el mundo. Allí, la ingesta de gaseosas se duplicó en 7 años y el sobrepeso y la obesidad alcanzaron a tres cuartos de la población adulta y al $25 \%$ de los niños 9 .

Entre los principales mecanismos por los cuales las bebidas azucaradas promueven el sobrepeso y la obesidad e incrementan los factores de riesgo cardiovascular se destaca el aumento del contenido calórico, la estimulación del apetito y los efectos adversos al consumo de jarabe de maíz alto en fructosa. Sobre este último aspecto, varios trabajos han mostrado que el exceso de fructosa promueve la «lipogénesis» de novo hepática a través de la síntesis de triglicéridos hepáticos ${ }^{10,11}$. La sobreproducción de estos contribuiría a un incremento del tejido adiposo y a la posterior acumulación ectópica de lípidos, fenómeno conocido también como «lipotoxicidad», la cual lleva a una resistencia a la insulina ${ }^{11}$. La fructosa puede, además, aumentar el ácido úrico en suero, reduciendo la sintasa de óxido nítrico endotelial, con la consecuente disfunción endotelial-mecanismo asociado al accidente cerebrovascular isquémico hemorrágico y a la presencia de DM-2-12. Es importante anotar que la obesidad es un factor de riesgo para DM-2, aunque no es el único mediador en la asociación entre la ingesta de bebidas azucaradas y esta, pues se han descrito mecanismos relacionados con el índice glucémico de las bebidas azucaradas y la consecuente resistencia a la insulina ${ }^{1}$.

Dentro de la misma problemática, se ha demostrado relación entre la ingesta habitual de bebidas azucaradas y el aumento de triglicéridos y colesterol unido a lipoproteínas de baja densidad (c-LDL), y la disminución proporcional del colesterol unido a lipoproteínas de alta densidad (c$\mathrm{HDL}$ ), creando un perfil lipídico aterogénico ${ }^{13}$. Incluso se ha comprobado el efecto hipocalcemiante inmediato cuando existe un consumo de bebidas azucaradas carbonatadas superior a $1.500 \mathrm{~mL} / \mathrm{sem}$, con riesgo incrementado de fracturas óseas ${ }^{14}$.

El consumo excesivo de bebidas azucaradas, con edulcorantes y saborizantes, se ha convertido en parte de la dieta cotidiana de los colombianos, constituyendo una fuente 
importante de azúcar añadido y, por tanto, de aporte calórico total en la dieta ${ }^{15}$. Datos de la Encuesta Nacional de Situación Nutricional en Colombia $2010^{15}$ muestran que estos productos son consumidos por el $81,2 \%$ de los colombianos, donde el $22,1 \%$ lo incluye dentro de su alimentación diaria y casi el $50 \%$ lo hace con una frecuencia semanal. Hay que tener en cuenta que la alimentación es uno de los factores que determinan la salud de los individuos y que la población universitaria se ha catalogado como vulnerable desde el punto de vista nutricional, ya que están en la construcción de nuevos hábitos de vida influidos por la cultura, la familia, los medios de comunicación y la escasez de tiempo, que han resultado poco saludables debido al alto consumo de snacks, bebidas azucaradas y alcohol ${ }^{14,16}$. Como se mencionó, las bebidas azucaradas son una de las categorías de alimentos que acumulan más evidencia científica en su contra, lo cual plantea la necesidad de limitar su consumo con políticas públicas de impacto poblacional, que trasciendan acciones centradas exclusivamente en la responsabilidad individual ${ }^{17}$. En Colombia, la prevalencia de factores de riesgo muestra una variación regional, con una relación invertida con la ingesta de bebidas azucaradas. Sin embargo, existen pocos estudios comparativos de la distribución de factores de riesgo, si se tiene en cuenta el consumo regular de bebidas azucaradas.

\section{Objetivo}

Examinar si el consumo de bebidas azucaradas se relaciona con alteraciones en el perfil lipídico-metabólico y con marcadores de adiposidad en una muestra de estudiantes universitarios de Colombia.

\section{Materiales y métodos}

\section{Diseño y población}

Durante el primer semestre de 2014 se planteó un estudio descriptivo y transversal en 280 estudiantes universitarios $(73,9 \%$ varones; edad $23,3 \pm 2,3$ años; peso corporal $61,2 \pm 11,3 \mathrm{~kg}$; IMC 22,5 $\pm 3,6 \mathrm{~kg} / \mathrm{m}^{-1}$ ) procedentes de 3 universidades de Bogotá, Colombia. La selección de la muestra se realizó mediante convocatoria voluntaria. Se excluyeron participantes con diagnóstico médico o clínico de enfermedad sistémica mayor, como DM-2, hipertensión arterial, hipo/hipertiroidismo, antecedentes de historia de abuso de drogas o alcohol, IMC $\geq 30 \mathrm{~kg} / \mathrm{m}^{-1}$ y padecimiento de procesos inflamatorios o infecciosos. Se obtuvo el consentimiento informado por escrito de cada participante y el Comité de Ética en Humanos del centro académico aprobó la intervención, siguiendo las normas deontológicas reconocidas por la Declaración de Helsinki y la normativa legal vigente colombiana que regula la investigación en humanos (Resolución 008430 del Ministerio de Salud de Colombia, acta de aprobación N. ${ }^{\circ}$ 01-1802-2013). Los participantes que aceptaron y firmaron el consentimiento informado se citaron para los procedimientos que se describen a continuación.

\section{Medición antropométrica y clínica}

De cada participante se obtuvieron datos como: a) antecedentes familiares de enfermedad cardiovascular; b) encuesta de antecedentes personales, y c) valoración antropométrica: altura, peso, circunferencia de cintura y perímetro de cadera. Se tomaron mediante las técnicas estandarizadas de López et al. ${ }^{18}$ en población colombiana. La altura se registró en estiramiento con estadímetro portátil (SECA 206 ${ }^{\circledR}$; Hamburgo, Alemania) (rango 0-220 cm) de $1 \mathrm{~mm}$ de precisión. El peso se midió con balanza de piso Tanita (modelo TBF-410GS ${ }^{T M}$, Arlington Heights, IL, Estados Unidos) con capacidad máxima de $200 \mathrm{~kg}$ y mínima de $100 \mathrm{~g}$. Con estas variables se calculó el $\mathrm{IMC}$ en $\mathrm{kg} / \mathrm{m}^{-1}$. Posteriormente, con cinta métrica plástica con una precisión de $1 \mathrm{~mm}$ (Holtain Ltd., Crymych Dyfed, Reino Unido) se midió la circunferencia de cintura y el perímetro de cadera tomando los referentes anatómicos descritos por Frisancho ${ }^{19}$. El porcentaje de grasa se estimó con el equipo de impedancia bioeléctrica segmentada de 4 puntos táctiles de electrodos Tanita (modelo TBF-410GS ${ }^{\text {TM }}$, Arlington Heights, IL, Estados Unidos) de acuerdo con las indicaciones y ecuaciones señaladas en el manual del usuario. La frecuencia de inducción se valoró a una intensidad de $50 \mathrm{kHz}$, con una sensibilidad de estimación de la masa de grasa de $0,1 \mathrm{~kg}$ $(0,1 \%)$. La medición se realizó luego de $8-10 \mathrm{~h}$ de ayuno, con la vejiga vacía y sobre una superficie no conductora. El índice de adiposidad corporal se estimó con la ecuación propuesta por Bergman et al. ${ }^{20}$ :

$\mathrm{IAC}=\left[(\mathrm{PC}\right.$, en $\left.\left.\mathrm{cm}) /\left((\text { altura, en } \mathrm{m})^{1,5}\right)-18\right)\right]$.

\section{Consumo de bebidas azucaradas}

Se indagó por el tipo de bebidas azucaradas que consumieron con mayor frecuencia en los últimos 7 días, mediante el Brief Questionnaire to Assess Habitual Beverage Intake (BEVQ-15) ${ }^{21}$ en los dominios: a) bebidas gaseosas regulares tipo cola; b) bebidas gaseosas tipo dieta: zero o light, y c) refrescos tipo jugo envasados. Posteriormente, se identificó la frecuencia de consumo para cada dominio en 3 categorías: $350 \mathrm{~mL}$ o menos, 700 a $1.050 \mathrm{~mL}$ y más de $1.050 \mathrm{~mL}$.

\section{Mediciones bioquímicas}

Se realizaron a través de la toma de una muestra sanguínea obtenida según las recomendaciones técnicas del fabricante para colesterol total, triglicéridos y c-HDL mediante equipo portátil CardioChek ${ }^{\circledR}$. El c-LDL se calculó por la fórmula de Friedewald ${ }^{22}$ ajustada por las concentraciones séricas de triglicéridos. La glucemia capilar se determinó con glucómetro Accu-Chek ${ }^{\circledR}$ Performa siguiendo las especificaciones técnicas recomendadas por el fabricante. Las extracciones de sangre se realizaron entre las 08:00 y las 09:00 a. m., tras 8-10 h de ayuno. Se calculó el índice aterogénico o índice de Castelli con la razón colesterol total/c- $\mathrm{HDL}^{23}$. Con los valores de triglicéridos, c-LDL, C-HDL y glucosa se estableció un índice lipídico-metabólico de riesgo cardiovascular como se indica en el trabajo de García-Artero et al. ${ }^{24}$. Cada una de estas variables sanguíneas fue tipificada como Z = ([valor - media] / desviación estándar $)$. La variable tipificada del c-HDL fue multiplicada por [-1] debido a que su relación con el riesgo cardiovascular es contraria al resto de las variables. El índice lipídico-metabólico de riesgo cardiovascular se calculó como la suma de las 4 variables tipificadas, de modo que los valores inferiores 
Tabla 1 Características antropométricas y perfil lipídico-metabólico de la muestra $(n=280)$

\begin{tabular}{|c|c|c|c|}
\hline Característica & $\begin{array}{l}\text { Varones } \\
(n=207)\end{array}$ & $\begin{array}{c}\text { Mujeres } \\
(n=73)\end{array}$ & Valor $p$ \\
\hline Edad (años) & $21,1 \pm 2,9$ & $22,4 \pm 3,1$ & 0,339 \\
\hline Estatura (m) & $1,75 \pm 0,5$ & $1,60 \pm 0,4$ & 0,009 \\
\hline Peso corporal (kg) & $68,1 \pm 8,8$ & $55,8 \pm 8,3$ & 0,001 \\
\hline IMC $\left(\mathrm{kg} / \mathrm{m}^{-1}\right)$ & $22,8 \pm 2,9$ & $21,8 \pm 2,5$ & 0,010 \\
\hline Circunferencia de cintura $(\mathrm{cm})$ & $77,4 \pm 6,4$ & $69,7 \pm 6,1$ & 0,001 \\
\hline Porcentaje de grasa $(\%)$ & $12,3 \pm 5,1$ & $23,7 \pm 6,7$ & 0,001 \\
\hline Índice de adiposidad corporal (\%) & $23,3 \pm 2,7$ & $28,6 \pm 2,8$ & 0,001 \\
\hline Colesterol total (mg/dL) & $138,8 \pm 27,9$ & $164,1 \pm 34,6$ & 0,001 \\
\hline Triglicéridos (mg/dL) & $83,1 \pm 42,0$ & $83,6 \pm 38,6$ & 0,938 \\
\hline $\mathrm{c}-\mathrm{LDL}(\mathrm{mg} / \mathrm{dL})$ & $81,5 \pm 24,9$ & $95,1 \pm 26,5$ & 0,001 \\
\hline $\mathrm{c}-\mathrm{HDL}(\mathrm{mg} / \mathrm{dL})$ & $41,1 \pm 9,4$ & $51,4 \pm 11,5$ & 0,001 \\
\hline Glucosa (mg/dL) & $85,7 \pm 9,3$ & $76,6 \pm 8,3$ & 0,001 \\
\hline Índice de Castelli & $1,7 \pm 0,2$ & $1,7 \pm 0,4$ & 0,906 \\
\hline Índice arterial & $3,6 \pm 2,1$ & $3,2 \pm 0,6$ & 0,157 \\
\hline
\end{tabular}

c-HDL: colesterol unido a lipoproteínas de alta densidad; c-LDL: colesterol unido a lipoproteínas de baja densidad; IMC: índice de masa corporal.

a este parámetro suponen un perfil lipídico-metabólico más cardiosaludable. Por la definición, su media es cero.

\section{Marcadores de adiposidad}

Se tomaron los criterios de riesgo cardiovascular reportados en el Programa Nacional de Educación del Colesterol ${ }^{25}$, la Federación Internacional de Diabetes ${ }^{26}$ y el Departamento de Salud de los Estados Unidos ${ }^{25}$ : IMC $\geq 25,1 \mathrm{~kg} / \mathrm{m}^{-1}$, obesidad abdominal (circunferencia de cintura $\geq 88 \mathrm{~cm}$ en varones y $80 \mathrm{~cm}$ en mujeres), adiposidad (porcentaje de grasa e índice de adiposidad corporal $\geq 25$ ). Las dimensiones y medidas citadas se tomaron con dispositivos homologados y de acuerdo con las normas del programa biológico internacional, elaborado por el International Council of Scientific Unions, que recoge los procedimientos esenciales para el estudio biológico de las poblaciones humanas ${ }^{27}$. Mediante la ecuación: $n=\left(Z_{1-\alpha / 2}+Z_{1-\beta}\right)^{2}$ p0 $(1-p 0) / \delta^{2}$; $n=10,5(0,20) / 0,01$ se calculó un tamaño de muestra de 210 participantes, para obtener un $30 \%$ de prevalencia promedio de sobrepeso asociado al consumo de bebidas azucaradas en ambos sexos.

\section{Análisis estadístico}

El procesamiento y análisis de la información se realizó con el programa Statistical Package for Social Science ${ }^{\circledR}$ software, versión 18 (SPSS; Chicago, IL, Estados Unidos). Los valores continuos se expresaron como media y desviación estándar. Se aplicaron pruebas de homogeneidad de varianzas; cuando las pruebas estadísticas lo permitieron se utilizaron pruebas paramétricas ajustadas por sexo, edad, índice de masa corporal y circunferencia de cintura. El estadístico t se utilizó para observar diferencias entre las variables continuas, y el estadístico $\chi^{2}$ para las variables categóricas. La relación entre el consumo de bebidas azucaradas y el índice lipídico-metabólico se estableció usando un análisis de tendencia lineal mediante contraste polinómico.
El nivel de significación estadística se fijó a un valor $p<0,05$.

\section{Resultados}

\section{Análisis descriptivo}

En la tabla 1 se observa que el peso y la talla fueron mayores en los varones $(p=0,01)$, mientras que el índice de masa corporal fue similar en ambos sexos. El porcentaje de grasa estimado con el índice de adiposidad corporal y por impedancia bioeléctrica segmentada fue mayor en el grupo de mujeres $(p<0,01)$. Los parámetros bioquímicos revelaron valores más altos de colesterol total, c-LDL, c-HDL y glucosa $(p=0,001)$ en los varones, mientras que los niveles de triglicéridos, el índice de Castelli y el índice arterial fueron similares en ambos grupos.

\section{Análisis del consumo de bebidas azucaradas en varones y mujeres}

En la fig. 1 se observa que los varones dijeron consumir con menor frecuencia y cantidad en los últimos s7 días bebidas tipo cola (37,0 vs. $43,8 \%$ ), bebidas gaseosas tipo dieta ( 21,9 vs. $26,1 \%$ y jugos envasados $(35,0$ vs. $36,8 \%)$ que las mujeres $\left(x^{2}=0,337\right)$. Tampoco se advirtieron diferencias significativas respecto al patrón de ingesta de bebidas azucaradas gaseosas tipo dieta $\left(\chi^{2}=1,978\right)$ y/o refrescos envasados $\left(\chi^{2}=0,205\right)$ entre varones y mujeres.

\section{Relación entre consumo de bebidas azucaradas, índice lipídico-metabólico y marcadores de adiposidad en varones y mujeres}

En el grupo de varones, en aquellos que acusaron mayor consumo de bebidas azucaradas (+4 veces/sem), la circunferencia de cintura y el porcentaje de grasa fueron superiores y arrojaron mayores valores de colesterol total, 


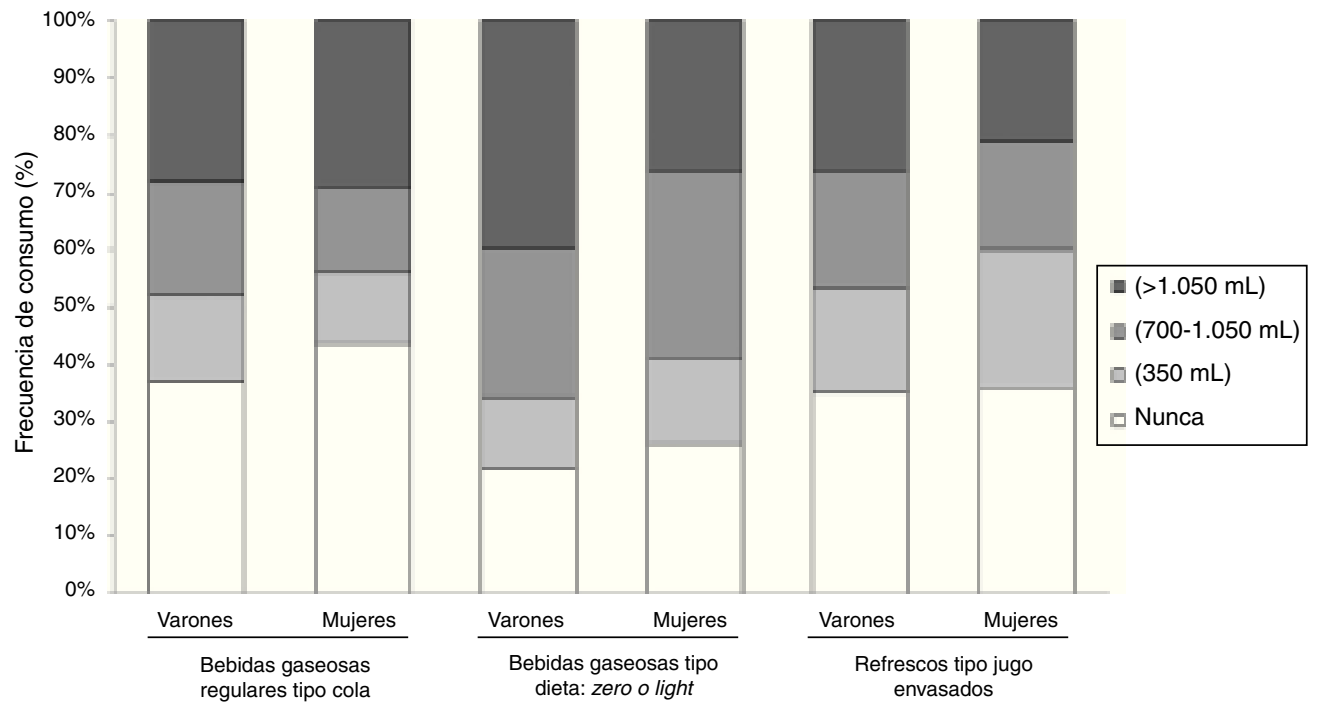

Figura 1 Análisis del consumo de bebidas azucaradas en varones y mujeres.

triglicéridos, $c-L D L$, e índices de Castelli y arterial ( $p>0,05)$. En mujeres, esta relación se observó en el índice de masa corporal ( $p$ tendencia $<0,05$ ), la circunferencia de cintura $y$ el porcentaje de grasa ( $p$ tendencia $<0,05)$. Además, mostraron menor valor en los niveles de triglicéridos y c-LDL al compararlos con los de los participantes que respondieron «nunca» consumir en los últimos 7 días bebidas azucaradas ( $\mathrm{p}$ tendencia $<0,05)($ tabla 2$)$.

Tabla 2 Clusters de bebidas azucaradas y su relación con el índice lipídico-metabólico y marcadores de adiposidad según sexo

\begin{tabular}{|c|c|c|c|c|c|}
\hline \multirow[t]{2}{*}{ Característica } & \multicolumn{5}{|c|}{ Frecuencia de consumo (semanal) } \\
\hline & Nunca & $1(350 \mathrm{~mL})$ & $2-3(700-1.050 \mathrm{~mL})$ & $+4(>1.050 \mathrm{~mL})$ & $p$ (tendencia) \\
\hline \multicolumn{6}{|l|}{ Varones $(n=207)$} \\
\hline IMC $\left(\mathrm{kg} / \mathrm{m}^{-1}\right)$ & $22,7 \pm 2,7$ & $22,5 \pm 2,7$ & $22,3 \pm 3,8$ & $22,6 \pm 2,8$ & 0,710 \\
\hline Circunferencia de cintura $(\mathrm{cm})$ & $75,7 \pm 6,9$ & $75,8 \pm 6,6$ & $75,8 \pm 7,1$ & $77,5 \pm 9,3$ & 0,511 \\
\hline Porcentaje de grasa (\%) & $12,9 \pm 7,0$ & $14,7 \pm 7,3$ & $14,8 \pm 7,1$ & $15,1 \pm 7,1$ & 0,400 \\
\hline IAC (\%) & $22,9 \pm 3,6$ & $24,4 \pm 3,5$ & $24,5 \pm 3,4$ & $24,8 \pm 3,7$ & 0,087 \\
\hline Colesterol total (mg/dL) & $137,9 \pm 28,1$ & $137,7 \pm 26,0$ & $138,8 \pm 27,9$ & $149,0 \pm 30,8$ & 0,361 \\
\hline Triglicéridos (mg/dL) & $80,8 \pm 40,8$ & $83,1 \pm 42,0$ & $87,2 \pm 40,7$ & $91,6 \pm 54,2$ & 0,543 \\
\hline $\mathrm{c}-\mathrm{LDL}(\mathrm{mg} / \mathrm{dL})$ & $77,8 \pm 22,7$ & $81,2 \pm 24,9$ & $81,5 \pm 24,9$ & $92,7 \pm 28,2$ & 0,165 \\
\hline $\mathrm{c}-\mathrm{HDL}(\mathrm{mg} / \mathrm{dL})$ & $42,5 \pm 10,0$ & $41,6 \pm 9,5$ & $41,4 \pm 9,4$ & $37,9 \pm 7,0$ & 0,165 \\
\hline Glucosa (mg/dL) & $83,4 \pm 10,3$ & $85,7 \pm 9,3$ & $86,1 \pm 8,9$ & $87,8 \pm 9,7$ & 0,283 \\
\hline Índice de Castelli & $1,65 \pm 0,19$ & $1,77 \pm 0,30$ & $1,77 \pm 0,28$ & $1,82 \pm 0,24$ & 0,171 \\
\hline Índice arterial & $3,4 \pm 1,0$ & $3,7 \pm 2,4$ & $3,7 \pm 2,1$ & $4,1 \pm 1,4$ & 0,573 \\
\hline \multicolumn{6}{|l|}{ Mujeres $(n=73)$} \\
\hline IMC $\left(\mathrm{kg} / \mathrm{m}^{-1}\right)$ & $20,5 \pm 2,5$ & $21,3 \pm 2,2$ & $21,2 \pm 1,7$ & $23,0 \pm 2,7$ & 0,021 \\
\hline Circunferencia de cintura $(\mathrm{cm})$ & $67,2 \pm 4,3$ & $68,6 \pm 6,0$ & $68,2 \pm 5,0$ & $72,2 \pm 6,8$ & 0,076 \\
\hline Porcentaje de grasa (\%) & $22,5 \pm 6,8$ & $23,5 \pm 6,7$ & $24,2 \pm 7,3$ & $24,4 \pm 5,6$ & 0,886 \\
\hline IAC (\%) & $27,2 \pm 3,2$ & $28,8 \pm 1,9$ & $28,8 \pm 2,2$ & $29,1 \pm 3,2$ & 0,294 \\
\hline Colesterol total (mg/dL) & $154,0 \pm 29,0$ & $159,1 \pm 28,8$ & $165,1 \pm 42,2$ & $182,5 \pm 36,8$ & 0,129 \\
\hline Triglicéridos (mg/dL) & $72,3 \pm 18,2$ & $80,0 \pm 40,2$ & $81,5 \pm 28,8$ & $105,9 \pm 56,3$ & 0,028 \\
\hline $\mathrm{c}-\mathrm{LDL}(\mathrm{mg} / \mathrm{dL})$ & $88,0 \pm 26,1$ & $88,2 \pm 18,8$ & $102,2 \pm 29,8$ & $105,8 \pm 27,2$ & 0,050 \\
\hline $\mathrm{c}-\mathrm{HDL}(\mathrm{mg} / \mathrm{dL})$ & $57,3 \pm 8,2$ & $50,0 \pm 12,1$ & $48,4 \pm 15,7$ & $49,8 \pm 7,9$ & 0,238 \\
\hline Glucosa (mg/dL) & $74,3 \pm 7,3$ & $75,3 \pm 7,0$ & $78,5 \pm 8,8$ & $77,1 \pm 10,1$ & 0,603 \\
\hline Índice de Castelli & $1,63 \pm 0,11$ & $1,76 \pm 0,20$ & $1,82 \pm 0,18$ & $1,83 \pm 0,38$ & 0,319 \\
\hline Índice arterial & $3,0 \pm 0,5$ & $3,2 \pm 1,1$ & $3,2 \pm 0,3$ & $3,5 \pm 0,5$ & 0,578 \\
\hline
\end{tabular}

c-HDL: colesterol unido a lipoproteínas de alta densidad; c-LDL: colesterol unido a lipoproteínas de baja densidad; IAC: índice de adiposidad corporal; IMC: índice de masa corporal.

a El cluster de bebidas azucaradas incluye la intersección de sujetos que consumen bebidas gaseosas regulares + tipo dieta + refrescos envasados. 


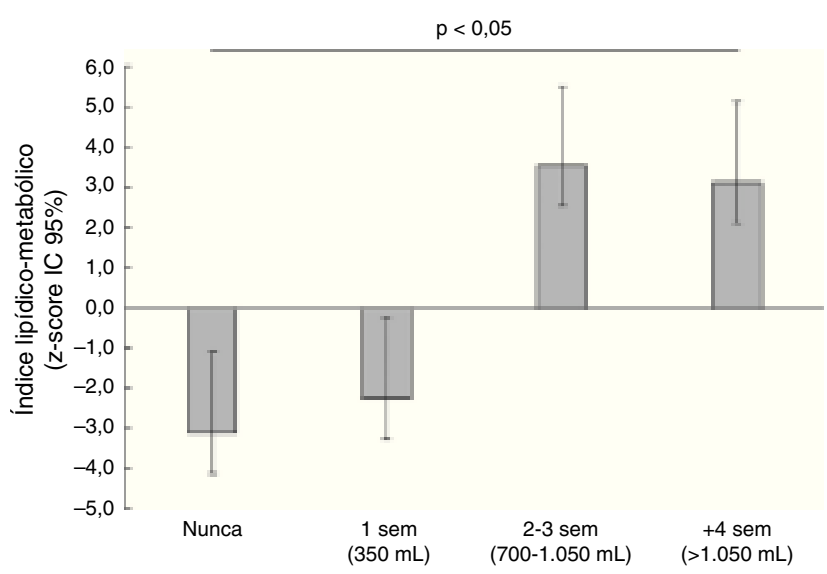

Figura 2 Relación entre el consumo de bebidas azucaradas y el índice lipídico-metabólico de riesgo cardiovascular en varones y mujeres.

Las barras de error representan los intervalos de confianza del 95\% (IC 95\%).

\section{Relación del índice lipídico-metabólico con el consumo de bebidas azucaradas}

El consumo de bebidas azucaradas mostró una relación significativa con el índice lipídico-metabólico. Las categorías más altas de consumo $(2-3$ y +4 veces/sem $)$ se relacionaron inversamente con el índice lipídico-metabólico $(p<0,05)$ tras ajustar por sexo, edad e índice de masa corporal (fig. 2).

\section{Discusión}

Este trabajo muestra que los estudiantes que presentaron mayor patrón de ingesta en el consumo de bebidas azucaradas tienen un perfil lipídico-metabólico elevado y, así mismo, mayores niveles en los marcadores de adiposidad estudiados. Aunque desde hace años se conoce la asociación entre el consumo de bebidas azucaradas -entre las que se incluyen la mayoría de las gaseosas, los refrescos procesados y los jugos a base de frutas-y el riesgo de enfermedades no transmisibles, ha sido en los últimos 15 años cuando se ha fijado la atención como una problemática de salud pública ${ }^{5,17,28}$.

El hallazgo hecho en nuestro trabajo acerca de una relación directa entre el consumo de bebidas azucaradas y el incremento en el riesgo cardiovascular respalda la hipótesis de otros estudios que han descrito que el consumo de estas bebidas se asocia con la aparición de DM-2, obesidad y síndrome metabólico. Las diferencias escasas entre la frecuencia de ingesta de bebidas azucaradas y los marcadores de adiposidad y en el perfil cardiometabólico constituyen una situación esperada y congruente con lo descrito en otros estudios similares cuando se trata de analizar factores que influyen en el desarrollo de una condición de riesgo multicausal.

Se ha especulado también que los refrescos embotellados promueven la obesidad mediante un desplazamiento de la energía que proviene de los alimentos sólidos de la dieta $^{29}$. En universitarios de Norteamérica se ha observado que el consumo de bebidas azucaradas se asocia con sobrepeso y que cada ración adicional $(360 \mathrm{~mL})$ incrementa en un $60 \%$ el riesgo de obesidad ${ }^{30}$. Esto coincide con reportes observacionales en niños y jóvenes estadounidenses ${ }^{3}$ y finlandeses ${ }^{31}$. Ludwig et al. ${ }^{32}$ muestran que con cada $200 \mathrm{~mL} /$ día de bebidas azucaradas consumidas, el riesgo de obesidad aumenta 1,6 veces y el IMC en $0,24 \mathrm{~kg} / \mathrm{m}^{-1}$; entre tanto, Malik et al. ${ }^{33}$, en un metaanálisis que incluyó 4 estudios de cohortes prospectivas, mostraron una asociación positiva entre el consumo de bebidas azucaradas y la elevación de lípidos y marcadores de obesidad en niños y adolescentes.

La evidencia descrita en humanos se ha explicado también en trabajos con modelos animales. Estudios en ratas, a las que se les reemplazó el agua por una solución con sacarosa al 8\%, mostraron incrementos en la presión arterial, menor sensibilidad a la insulina y ganancia de peso luego de 30 días de seguimiento ${ }^{34}$. Se ha descrito que el consumo de fructosa en animales incrementa los niveles de epinefrina en el corazón, el páncreas y el hígado ${ }^{35}$, lo que evidencia una mayor activación simpática en estos órganos a consecuencia de la activación del sistema reninaangiotensina-aldosterona, vía sistema nervioso simpático ${ }^{34}$.

Por otra parte, es preciso tener en cuenta algunos aspectos como limitantes del estudio. Por ejemplo, el tamaño de la muestra, las características propias de la población, el diseño del trabajo y el tipo de muestreo pueden ser considerados fuentes potenciales de sesgos. Tampoco fueron incluidas otras variables que pueden estar asociadas con el perfil de riesgo lipídico-metabólico y de obesidad, tales como la etnia, aspectos socioeconómicos, nutricionales, sociales y niveles de actividad física. Sin embargo, se observó convergencia de los resultados con datos reportados en otros estudios nacionales e internacionales ${ }^{2,3,14,29,30,32}$.

No obstante, la investigación sobre la influencia de la ingesta de bebidas azucaradas en el desarrollo de la enfermedad cardiometabólica es relativamente reciente. Estudios epidemiológicos como el actual, en el que se tienen en cuenta diferentes variables relacionadas con las enfermedades no transmisibles, son necesarios para establecer hipótesis que luego deberán confirmarse con otro tipo de diseños controlados, prospectivos y con la inclusión de medidas bioquímicas y de comportamiento, entre otras.

Pese a lo anterior, nuestros hallazgos brindan información que permitirá en los próximos años constatar y evaluar la trascendencia clínica de la relación encontrada en estudios epidemiológicos transversales, y la inclusión del consumo de bebidas azucaradas como un factor asociado con el riesgo de obesidad o de dislipidemia.

En conclusión, y a la luz de los resultados, el incremento en el consumo de bebidas azucaradas se relacionó con un mayor perfil lipídico-metabólico y con marcadores de adiposidad elevados en universitarios colombianos. Los datos obtenidos plantean la necesidad de limitar el consumo de bebidas azucaradas mediante un trabajo conjunto con autoridades gubernamentales, productores, publicistas, medios de comunicación y sociedad en general.

\section{Responsabilidades éticas}

Protección de personas y animales. Los autores declaran que los procedimientos seguidos se ajustaron a las normas éticas del comité de experimentación humana responsable y 
de acuerdo con la Asociación Médica Mundial y la Declaración de Helsinki.

Confidencialidad de los datos. Los autores declaran que han seguido los protocolos de su centro de trabajo sobre la publicación de datos de pacientes.

Derecho a la privacidad y consentimiento informado. Los autores han obtenido el consentimiento informado de los pacientes y/o sujetos referidos en el artículo. Este documento obra en poder del autor de correspondencia.

\section{Financiación}

Estos resultados forman parte del proyecto «Asociación de la fuerza prensil con manifestaciones tempranas de riesgo cardiovascular en adultos jóvenes colombianos», aprobado en la convocatoria nacional para jóvenes investigadores e innovadores COLCIENCIAS N. ${ }^{\circ}$ 617-2013 y la Vicerrectoría de Investigaciones convocatoria FODEIN-USTA 2014 N. ${ }^{\circ}$ 2013004.

\section{Conflicto de intereses}

Los autores declaran no tener conflictos de interés.

\section{Bibliografía}

1. Silva OP, Durán AS. Bebidas azucaradas, más que un simple refresco. Rev Chil Nutr. 2014;41:90-7.

2. Fagherazzi G, Vilier A, Saes Sartorelli D, Lajous M, Balkau B, Clavel-Chapelon F. Consumption of artificially and sugarsweetened beverages and incident type 2 diabetes in the Etude Epidemiologique aupres des femmes de la Mutuelle Generale de l'Education Nationale-European Prospective Investigation into Cancer and Nutrition cohort. Am J Clin Nutr. 2013;97:517-23.

3. Chan TF, Lin WT, Huang HL, Lee CY, Wu PW, Chiu YW, et al. Consumption of sugar-sweetened beverages is associated with components of the metabolic syndrome in adolescents. Nutrients. 2014;6:2088-103.

4. Malik AH, Akram Y, Shetty S, Malik SS, Yanchou Njike V. Impact of sugar-sweetened beverages on blood pressure. Am J Cardiol. 2014;113:1574-80.

5. Huang C, Huang J, Tian Y, Yang X, Gu D. Sugar sweetened beverages consumption and risk of coronary heart disease: A meta-analysis of prospective studies. Atherosclerosis. 2014;234:11-6.

6. Boyle P, Koechlin A, Autier P. Sweetened carbonated beverage consumption and cancer risk: Meta-analysis and review. Eur J Cancer Prev. 2014;23.

7. Rippe JM. The metabolic and endocrine response and health implications of consuming sugar-sweetened beverages: Findings from recent randomized controlled trials. Adv Nutr. 2013;4:677-86.

8. Malik VS, Popkin BM, Bray GA, Despres JP, Willett WC, Hu FB. Sugar-sweetened beverages and risk of metabolic syndrome and type 2 diabetes: A meta-analysis. Diabetes Care. 2010;33:2477-83.

9. Olaiz-Fernández G, Rivera-Dommarco J, Shamah-Levy T, Rojas R, Villalpando-Hernández S, Hernández-Avila M, et al. Encuesta Nacional de Salud y Nutrición 2006. Cuernavaca, México: Instituto Nacional de Salud Pública; 2006.
10. Bray GA. Energy and fructose from beverages sweetened with sugar or high-fructose corn syrup pose a health risk for some people. Adv Nutr. 2013;4:220-5.

11. Johnson RJ, Segal MS, Sautin Y, Nakagawa T, Feig DI, Kang $\mathrm{DH}$, et al. Potential role of sugar (fructose) in the epidemic of hypertension, obesity and the metabolic syndrome, diabetes, kidney disease, and cardiovascular disease. Am J Clin Nutr. 2007;86:899-906.

12. Johnson RJ, Perez-Pozo SE, Sautin YY, Manitius J, SanchezLozada LG, Feig DI, et al. Hypothesis: Could excessive fructose intake and uric acid cause type 2 diabetes? Endocr Rev. 2009;30:96-116.

13. Stanhope KL, Schwarz JM, Havel PJ. Adverse metabolic effects of dietary fructose: Results from the recent epidemiological, clinical, and mechanistic studies. Curr Opin Lipidol. 2013;24:198-206.

14. Mazariegos E, Guerrero-Romero F, Rodríguez M. Consumption of soft drinks with phosphoric acid as a risk factor for the development of hypocalcemia in children: A case-control study. J Pediatrics. 1995;126:940-2.

15. Instituto Colombiano de Bienestar Familiar (ICBF). Encuesta Nacional de la Situación Nutricional en Colombia (ENSIN). Bogotá: ICBF; 2010.

16. Triviño-Quintero LP, Dosman-González VA, Uribe-Vélez YL, Agredo-Zúñiga RA, Jerez-Valderrama AM, Ramírez Vélez R. Estudio del estilo de vida y su relación con factores de riesgo cardiovascular en adultos de mediana edad. Acta Med Colomb. 2009;34:158-63.

17. Gómez L, Jacoby E, Ibarra L, Lucumí D, Hernández A, Parra D, et al. Patrocinio de programas de actividad física por parte de la industria de bebidas azucaradas: ¿salud pública o relaciones públicas? Rev Saude Publica. 2011;45:423-7.

18. López CA, Ramírez-Vélez R, Gallardo CEG, Marmolejo LC. Características morfofuncionales de individuos físicamente activos. latreia. 2008;21:121-8.

19. Frisancho R. Anthropometric standard for the assessment of growth and nutritional status. Chapter II: Methods and materials. Ann Arbor: The University of Michigan Press; 1993. p. 9-31.

20. Bergman RN, Stefanovski D, Buchanan TA, Sumner AE, Reynolds JC, Sebring NG, et al. A better index of body adiposity. Obesity (Silver Spring). 2011;19:1083-9.

21. Hedrick VE, Savla J, Comber DL, Flack KD, Estabrooks PA, NsiahKumi PA, et al. Development of a brief questionnaire to assess habitual beverage intake (BEVQ-15): Sugar-sweetened beverages and total beverage energy intake. J Acad Nutr Diet. 2012;112:840-9.

22. Friedewald WT, Levy RI, Fredrickson DS. Estimation of the concentration of low-density lipoprotein cholesterol in plasma, without use of the preparative ultracentrifuge. Clin Chem. 1972;18:499-502.

23. Millán J, Pintó X, Muñoz A, Zúñiga M, Rubiés-Prat J, Pallardo LF, et al. Lipoprotein ratios: Physiological significance and clinical usefulness in cardiovascular prevention. Vasc Health Risk Manag. 2009;5:757-65.

24. García-Artero E, Ortega FB, Ruiz JR, Mesa JL, Delgado M, González-Gross M, et al. Lipid and metabolic profiles in adolescents are affected more by physical fitness than physical activity (AVENA study). Rev Esp Cardiol. 2007;60:581-658.

25. Expert Panel on Detection, Evaluation, and Treatment of High Blood Cholesterol in Adults. Executive Summary of the Third Report of The National Cholesterol Education Program (NCEP) Expert Panel on Detection, Evaluation, and Treatment of High Blood Cholesterol in Adults (Adult Treatment Panel III). JAMA. 2001;285:2486-97.

26. International Diabetes Federation. Bruselas International Diabetes Federation; 2005. [consultado 12 Nov 2013]. Disponible en: http://www.eatlas.idf.org/Prevalence/index.cfm 
27. Weiner JS, Lourie JA. Practical human biology. 1st ed. London: Academic Press; 1981. p. 56.

28. Bray GA, Popkin BM. Dietary sugar and body weight: Have we reached a crisis in the epidemic of obesity and diabetes?: Health be damned! Pour on the sugar. Diabetes Care. 2014;37:950-6.

29. Gutiérrez-Ruvalcaba CL, Vásquez-Garibay $E$, Romero-Velarde $E$, Troyo-Sanromán R, Cabrera-Pivaral C, Ramírez-Magaña O. Consumo de refrescos y riesgo de obesidad en adolescentes de Guadalajara, México. Bol Med Hosp Infant Mex. 2009;66:522-8.

30. Shah T, Purohit G, Nair SP, Patel B, Rawal Y, Shah RM. Assessment of obesity, overweight and its association with the fast food consumption in medical students. J Clin Diagn Res. 2014;8:CC05-7.

31. Nissinen K, Mikkilä V, Männistö S, Lahti-Koski M, Räsänen L, Viikari J, et al. Sweets and sugar-sweetened soft drink intake in childhood in relation to adult BMI and overweight. The
Cardiovascular Risk in Young Finns Study. Public Health Nutr. 2009;12:2018-26.

32. Ludwig DS, Peterson KE, Gortmaker SL. Relation between consumption of sugar-sweetened drinks and childhood obesity: A prospective, observational analysis. Lancet. 2001;357:505-8.

33. Malik VS, Schulze MB, Hu FB. Intake of sugar-sweetened beverages and weight gain: A systematic review. Am J Clin Nutr. 2006;84:274-88.

34. Preuss HG, Echard B, Bagchi D, Perricone NV. Comparing effects of carbohydrate $(\mathrm{CHO})$ blockers and trivalent chromium on $\mathrm{CHO}$ induced insulin resistance and elevated blood pressure in rats. J Am Coll Nutr. 2013;32:58-65.

35. Young JB, Landsberg L. Effect of diet and cold exposure on norepinephrine turnover in pancreas and liver. Am J Physiol. 1979;236:E524-33. 